\section{Indices of response bias in the same-different experiment}

\author{
R. JOHN IRWIN, MICHAEL J. HAUTUS, \\ and MARGARET A. FRANCIS \\ University of Auckland, Auckland, New Zealand
}

The same-different experiment is popular for assessing perceptual performance, including the performance of people with neuropsychological deficits. Although the measurement of accuracy with this experiment is now well understood, the measurement of response bias remains problematic. Reformulating the decision space for the experiment can yield new bias indices that are analogous to, but numerically different from, those of the more familiar yes-no experiment. Isobias curves that show how hit rate covaries with false alarm rate for constant bias but varying accuracy are presented for eight indices, and best-fitting parameters of the isobias functions are determined for a set of experimental data. The theoretical status of the bias indices and their relation to other formulations are reviewed.

Judging whether two things are the same or different is frequently called for, both in daily life and in the psychological laboratory. The study of prosopagnosia, a disorder in which people have difficulty recognizing faces, offers a neuropsychological example. One way to study this disorder is to ask patients whether two faces are the same or different. But because the same-different experiment is prone to response biases, care has to be taken in obtaining an index of accuracy. The theory of signal detectability has now been extended to the same-different experiment (see Macmillan \& Creelman, 1991) so that the bias-free index $d^{\prime}$ can be computed for this experiment too. The properties of this index and its advantages over seemingly more straightforward measures of accuracy, such as percentage correct, are well known.

Less well understood than accuracy in the same-different experiment is the measurement of its counterpart, response bias. In many experiments, bias is a nuisance factor that needs to be controlled or eliminated. Sometimes, however, the bias adopted by an observer is of interest in its own right and therefore requires measurement. This may be especially important in the study of prosopagnosia if, as Tarr, Gauthier, and Behrmann (1998) and Gauthier, Behrmann, and Tarr (1999) report, the disorder alters same-different biases for various classes of objects, not just for faces. If so, the disorder is not a deficit

We thank Jennifer Stillman for useful discussions about these ideas and David Noreen and an anonymous reviewer for valuable comments on an earlier version of the manuscript. Correspondence concerning this article should be addressed to R. J. Irwin, Department of Psychology, University of Auckland, Private Bag 92019, Auckland, New Zealand (e-mail: j.irwin@auckland.ac.nz). specific to faces. To draw such a conclusion from laboratory evidence, however, requires measuring response bias in the same-different experiment.

The importance of proper measures of bias for other designs is well recognized, and Snodgrass and Corwin (1988) have reviewed various indices of bias for recognition memory in the yes-no experiment. They observed that different diagnostic groups (e.g., Huntington's disease patients and amnesic patients) differed not only in recognition accuracy, but also in response bias. However, the indices of bias that they and Macmillan and Creelman (1990) reviewed for the yes-no experiment do not transpose to the same-different experiment. Macmillan and Creelman (1991) have developed special measures of response bias for the same-different design, but we provide another perspective on indices of bias for that task by reformulating its decision space.

\section{ELEMENTS OF THE SAME-DIFFERENT EXPERIMENT}

Before developing the isobias indices, we will summarize the elements of the same-different experiment and its detection-theoretic analysis. (Macmillan \& Creelman, 1991, provide a full account of this design.) As with most psychophysical methods, performance is assessed from judgments made over a series of trials. In the samedifferent method, every trial contains two observation intervals (separated in time or space), and one of two events occurs in each interval. With two intervals and two events, there are four possible arrangements of the events in a trial. In the standard version, every arrangement is equally likely on any trial. After examining the two intervals, the observer reports whether the two events were the same or different. A detection-theoretic analysis of the judgments makes the standard assumption that the observations are distributed normally with equal variance.

\section{TWO DECISION STRATEGIES}

Whereas for the yes-no experiment there is but one reasonable strategy for deciding whether an event occurred in the observation interval, for the same-different experiment there are at least two strategies available to an observer (Macmillan \& Creelman, 1991). One rule for solving the same-different problem is to take the difference between the observations of a trial, and if that difference exceeds a criterion magnitude, the events are declared different. This is known as the differencing strategy. An alternative policy is to consider each observation independently and to assign each to one or the other event so that the two events can then be declared the same or different. This rule is known as the independentobservations strategy. Analysis shows that the two strategies lead to different levels of accuracy and to different 
shaped receiver-operating characteristics (ROCs). The independent-observations strategy is always equal or superior to the differencing strategy (see, e.g., Hautus, Irwin, \& Sutherland, 1994; Irwin, Hautus, \& Butcher, 1999).

\section{VARIETIES OF BIAS INDICES FOR THE SAME-DIFFERENT EXPERIMENT}

Specification of response bias in the same-different experiment, as in other designs, falls into two classes. One class specifies the criterion location or cutoff point on the decision variable, and the other class specifies a likelihood ratio. For any given model, these two specifications are related, although not necessarily monotonically. Even though criterion location and likelihood ratio are related, an observer who adopts a constant location for different levels of accuracy will have a variable likelihood ratio, and one who adopts a constant likelihood ratio will have a variable cutoff point.

The availability of two strategies for the observer in the same-different experiment complicates the analysis of response bias in the experiment. A further complication arises because the specification of criterion location on the decision axis can be made with respect to various reference points, such as the origin of the axis or the point corresponding to the neutral likelihood ratio of 1 . For the independent-observations strategy, the origin of the decision axis can be placed at the point corresponding to a likelihood ratio of 1 , just as it can be in the yes-no experiment. But for the differencing strategy, a likelihood ratio of 1 does not coincide with the origin of the decision axis. Still another choice available is to specify the degree of bias relative to the observer's accuracy (see Macmillan \& Creelman, 1991). There are at least eight indices of bias for the same-different experiment: five for differencing, and three for independent observations. Four of the differencing indices specify a location on the decision axis, and the fifth specifies a likelihood ratio; two of the independent-observations indices specify a location, and the third specifies a likelihood ratio. Macmillan and Creelman (1996) have noted that every bias index implies its own isobias curve: the curve showing how hit and false alarm rates covary as a function of discriminability when bias remains constant. We therefore also consider the isobias functions for the eight indices we review.

\section{The Decision Space for the Differencing Strategy}

Figure 1 illustrates the decision space for the differencing strategy; there are two probability density functions, one for same events and one for different events. The equations for these densities can be derived by differentiating the equations for the hit rate and the false alarm rate, ${ }_{1}$ reported elsewhere (e.g., Hautus et al., 1994, Equations 1 and 2). Even though the events are assumed to be Gaussian in distribution, their differences are not Gauss- ian, because it is the absolute difference, rather than the signed difference, that serves as the decision variable. The densities in the example in Figure 1 are for events separated by a $d^{\prime}$ of 2 . This strategy leads to an asymmetrical ROC, as is shown in the insert in the figure.

Five indices of response bias are illustrated in Figure 1. Two numerical examples of each, together with their locations on the ROC, are shown. One index, $k$, is the distance from the origin of the decision axis. An observer who holds this index of bias constant for different levels of accuracy sets the criterion at a fixed point on the decision axis. A second way of designating criterion location is in terms of its distance from the point corresponding to a likelihood ratio of 1 , the point at which the two densities intersect. This index is designated $c_{d}$. An observer who holds this index constant locates the criterion at a constant distance from the point representing equal likelihood of same and different events. A third index expresses $c_{d}$ relative to accuracy. Following Macmillan and Creelman (1991), we use a prime to distinguish this relative index from its cousin, so that $c_{d}^{\prime}=c_{d} / d^{\prime}$. A fourth index, $c_{s d}$, was developed for the differencing strategy by Macmillan and Creelman (1991, Equation 6.5); it represents a constant distance on the decision axis from the point corresponding to $d^{\prime} / 2$. The fifth index, $\beta_{d}$, is the likelihood ratio itself. This index specifies the ratio of the likelihood of the events being the same to the likelihood of their being different and possesses some wellknown properties (see, e.g., Green \& Swets, 1966). An observer who holds the likelihood ratio constant must adjust the cutoff point as the distance between the two density functions, and, therefore, the observer's accuracy changes.

\section{Five Families of Isobias Functions for the Differencing Strategy}

Isobias functions for $\boldsymbol{k}$. The hit rate and the false alarm rate are given by the definite integral of each density function between zero and the criterion, $k$. (Note that the hit rate and the false alarm rate for this representation are therefore given by the areas of the density functions to the left of the criterion, not to the right, as is usually the case.) Isobias functions can then be derived by determining how the hit rate varies with the false alarm rate for a given criterion as a function of $d^{\prime}$. The parametric equations for the differencing rule are given in Equation $\mathrm{A} 1$ of the Appendix. These equations are able to serve as the parametric equations for all five isobias functions, because the indices for the differencing rule are related to each other. We have accommodated this by substituting appropriate expressions for the variable $k$ in Equation A1. Figure 2A illustrates examples of such isobias functions for $k$. Above the major diagonal, they turn out to be horizontal straight lines corresponding to a given hit rate. The lines are also shown below the diagonal for completeness (see Macmillan \& Creelman, 1990), but 


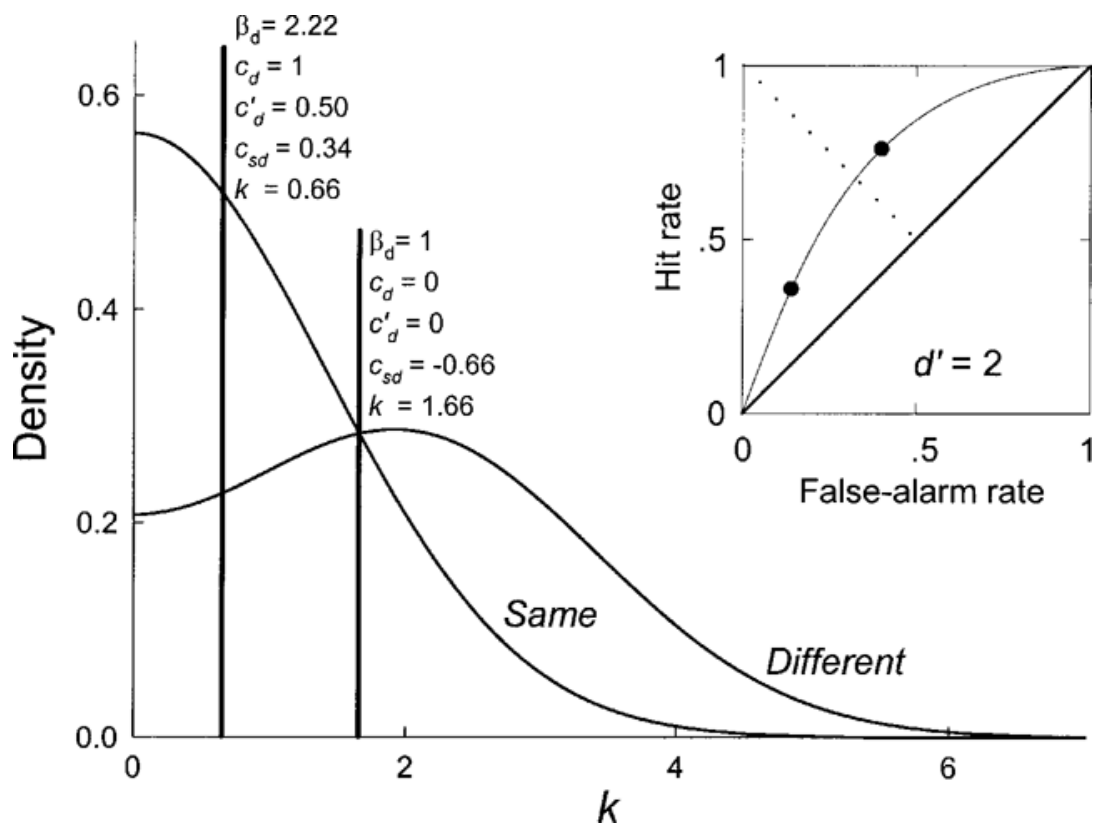

Figure 1. Probability density functions for the differencing strategy when $d^{\prime}=2$. The decision axis for the differencing strategy, $k$, is the absolute value of the difference between the two observations of a trial. Also illustrated are the likelihood ratio, $\beta_{d}$, and the criterion locations, $c_{d}, c_{d}^{\prime}$ and $k$, at the neutral likelihood-ratio point and at a second point. The corresponding ROC for $d^{\prime}=\mathbf{2}$ is also illustrated, with the location of the two values of isobias marked.

that region of the ROC square represents below-chance performance, and presumably data can fall there only through sampling errors.

Isobias functions for $\boldsymbol{c}_{\boldsymbol{d}}$. Next, consider the isobias functions of the differencing model for the index $c_{d}$. Again, the hit rate and the false alarm rate are found by integrating the density functions up to the criterion, $c_{d}$. Here, the appropriate substitution for $k$ in Equation A1 is given by Equation $\mathrm{A} 3$ in the Appendix. When that expression is substituted for $k$ in Equation A1, the parametric equations for $c_{d}$ are obtained. The resulting curves, illustrated in Figure 2C, are similar to the comparable curves for the yes-no experiment, except for an asymmetry about the negative diagonal of the ROC square.

Isobias functions for $c_{d}^{\prime}$. Isobias functions for the index $c_{d}^{\prime}\left(c_{d}\right.$ expressed relative to $\left.d^{\prime}\right)$ are illustrated in Figure 2D. The idea is that different values of $c_{d}$ represent different degrees of bias for different levels of accuracy. For this index, the functions are generated by the substitution of $c_{d}^{\prime} d^{\prime}$ for $c_{d}$ in Equation A3 of the Appendix, and the resulting expression for $k$, given in Equation A4, is substituted in Equation A1.

Isobias functions for $\boldsymbol{c}_{\boldsymbol{s} d}$. The isobias functions for $c_{s d}$, shown in Figure 2E, are based on Equation 6.5 of Macmillan and Creelman (1991). They were obtained by substituting in Equation A1 the expression for $k$ given in Equation A5 of the Appendix. Here, the location index is referenced with respect to the value corresponding to $d^{\prime} / 2$.
Isobias functions for $\beta_{d}$. To compute the isobias functions for $\beta_{d}$ requires substituting the appropriate expression, given in Equation A2 in the Appendix, for $k$ in Equation A1. Figure 2B illustrates the resulting isobias curves. Again, the curves resemble the comparable yes-no ones but are asymmetrical about the negative diagonal of the ROC square. (Because $c_{d}$ is specified as the distance from $\beta_{d}=1$, the asymmetry in the ROC square of $\beta_{d}$ implies the asymmetry of $c_{d}$.)

\section{The Decision Space for the \\ Independent-Observations Strategy}

Three indices, $c_{i}, c_{\mathrm{i}}^{\prime}$, and $\beta_{\mathrm{i}}$, comparable to those for the differencing strategy, are available for measuring response bias of an observer adopting the independentobservations strategy. (Irwin \& Hautus, 1997, provide a detailed description of the independent-observations model based on likelihood ratio.) In Figure 3, we have chosen to represent the decision axis for this strategy as $c_{i}$, with the criterion placed at $\ln (\beta) / d^{\prime}$ (see Irwin \& Hautus, 1997), because the independent-observations strategy can be likened to two separate yes-no decisions, one for each observation interval. In the yes-no experiment, the location of the criterion in units of standard deviation from the neutral bias point is given by $\ln (\beta) / d^{\prime}$ (Macmillan \& Creelman, 1991, Equation 2.10; Noreen, 1981, p. 246, Decision Rule 3). Figure 3 illustrates the probability densities for $d^{\prime}=2$ and the bias index $c_{i}$ on that de- 

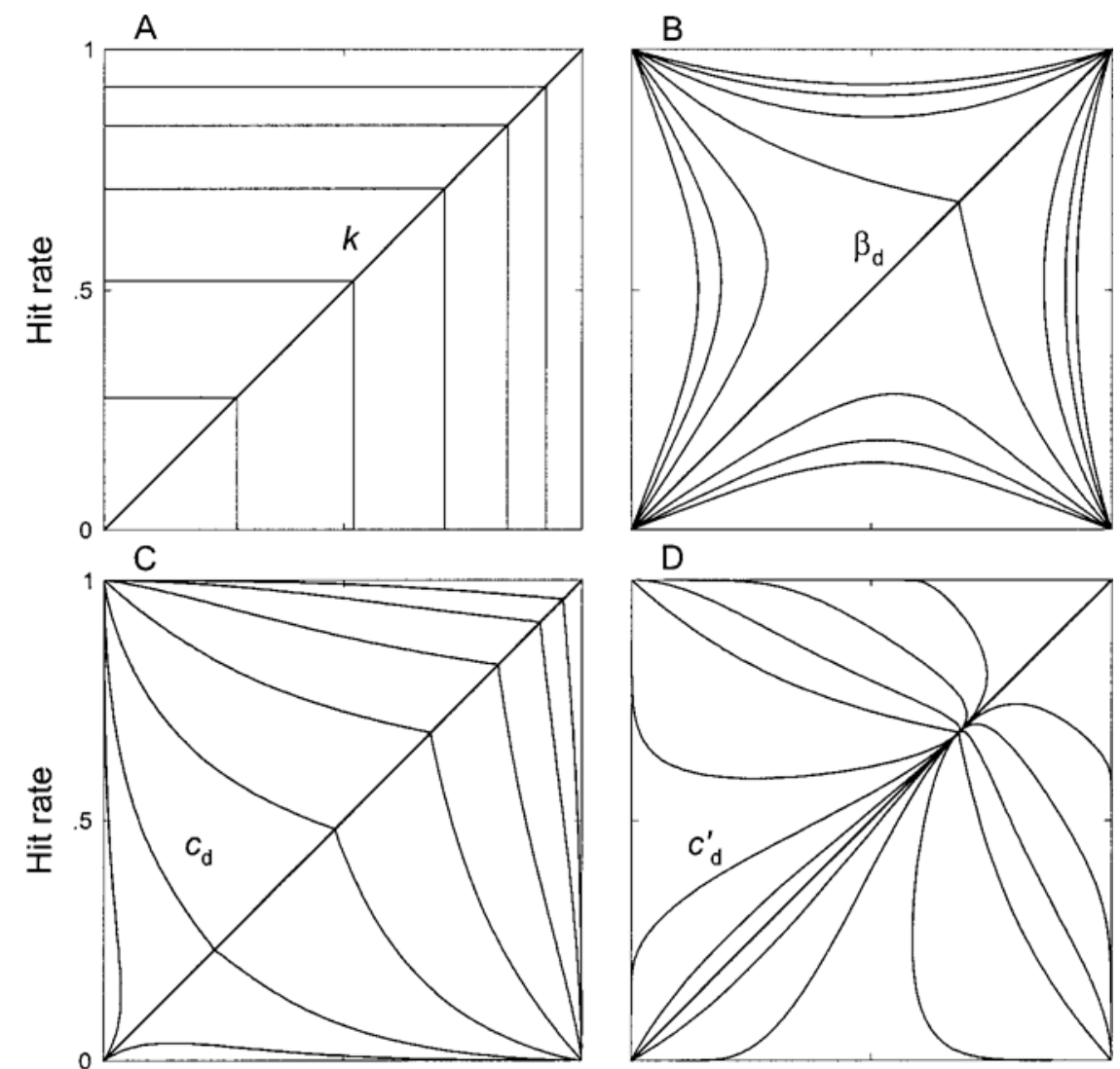

$\mathrm{D}$
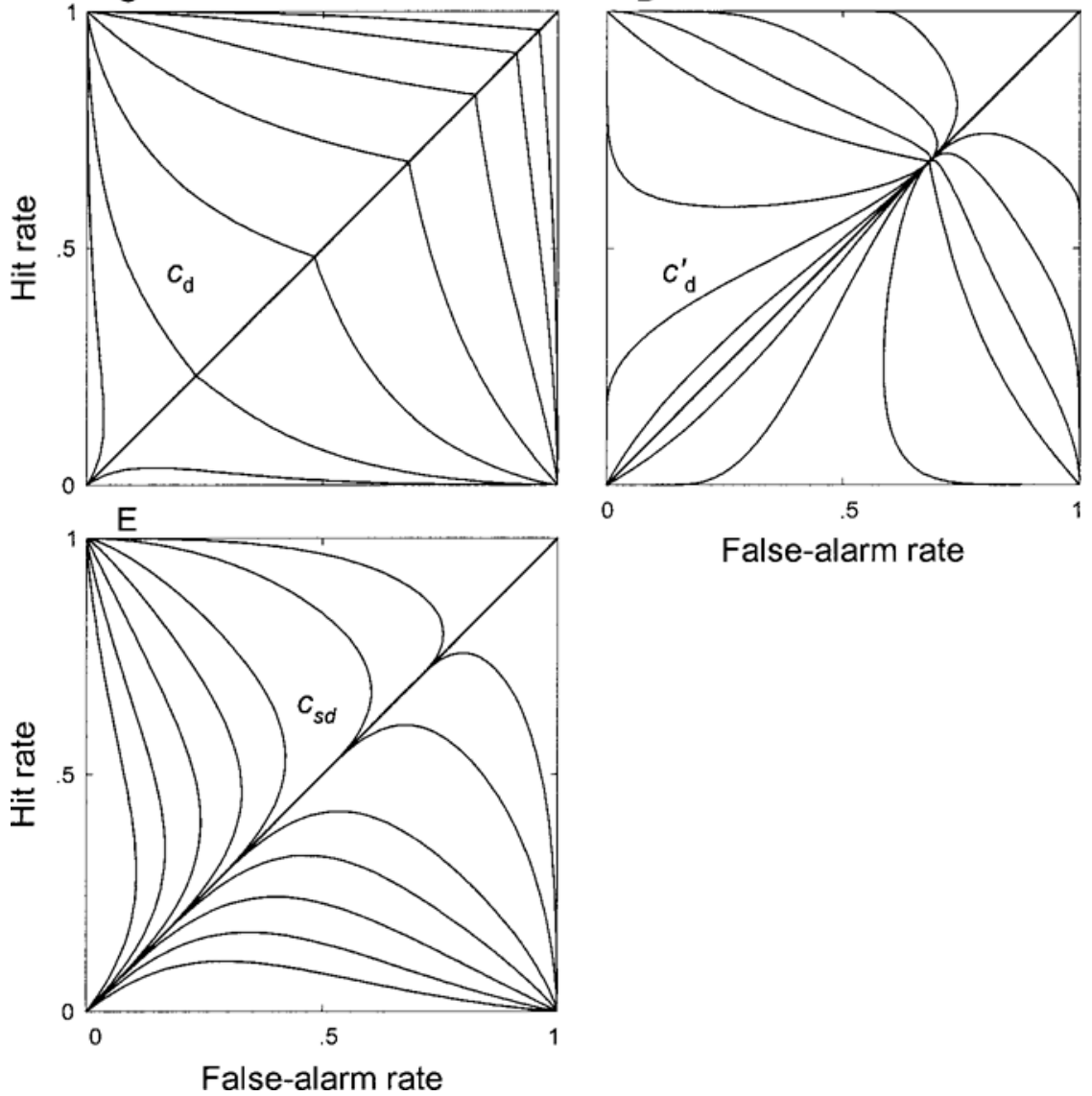

False-alarm rate

Figure 2. Isobias curves for the differencing strategy for $k(\mathrm{~A}), \beta_{d}(\mathrm{~B}), c_{d}(\mathrm{C}), c_{d}^{\prime}(\mathrm{D})$, and $c_{s d}(\mathrm{E})$. The values illustrated are $k=0.5,1.0,1.5,2.0$, and $2.5 ; \beta_{d}=2.5,2.0,1.5,1.0,0.67,0.5$, and 0.4 ; $c_{d}$ or $c_{d}^{\prime}=1.5,1.0,0.5,0.0,-0.5,-1.0$, and $-1.5 ;$ and $c_{s d}=0.5,0.25,0.0,-0.25,-0.5,-1.0$, and -1.5 . Theoretically, the curves continue below the major diagonal as shown, although in practice experimental data would be expected in that region by chance only. The below-chance functions may nevertheless be useful for fitting models when some data fall in that region.

cision axis. The corresponding symmetrical ROC for this case and the location on the ROC of the two criterion points are shown in the insert.

Adopting $c_{i}$ as the decision axis has three benefits: (1) It provides an index of criterion location that is comparable to one for the yes-no method, (2) ROCs based on its density functions are nearly identical to those based on the likelihood ratio, and (3) the equations for this version of the independent-observations strategy are substantially simpler than those based on $\beta_{\mathrm{i}}$ (Irwin \& Hautus, 1997). However, this transformation does not preserve the exact value of the likelihood ratio for the strategy, be- 


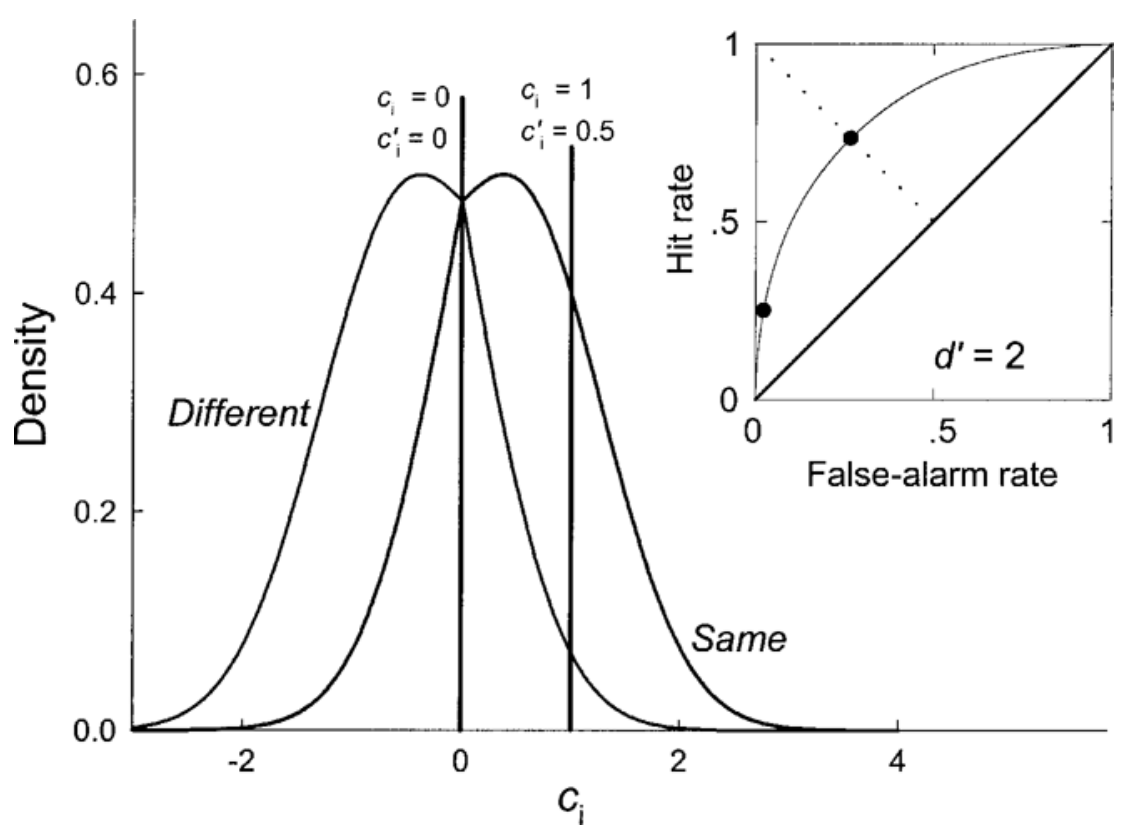

Figure 3. Probability density functions for the independent-observations strategy when $d^{\prime}=2$. The decision axis shown for this strategy is $c_{i}$. Two criterion values of $c_{i}$ and $c_{i}^{\prime}$ are illustrated. The corresponding ROC for $d^{\prime}=2$ is also illustrated, with the location of the two values of isobias marked.

cause the decision boundaries for each bias index are different, except for the neutral likelihood ratio of 1 , where the criterion based on $c_{i}$ is identical to that based on $\beta_{\mathrm{i}}$ (Irwin \& Hautus, 1997).

The relation between a decision criterion anchored at $\ln \left(\beta / d^{\prime}\right)$ and one based on $\beta$ can be seen in the twodimensional portrayal of the decision space for the samedifferent experiment (see Irwin \& Hautus, 1997, Figure 1$)$. For the case of neutral bias when $\beta=1$, criterion boundaries based on $\ln \left(\beta / d^{\prime}\right)$ and $\beta$ coincide and so partition the decision space identically; but when $\beta \neq 1$, criterion boundaries based on $\ln \left(\beta / d^{\prime}\right)$ and $\beta$ coincide only at extreme values of the decision space, so that they partition the decision space differently. Two yes-no decisions based on the criterion $\ln \left(\beta / d^{\prime}\right)$ constitute a conjunctive rule for the same-different task: In the two-dimensional representation, the observer accepts two observations as stemming from the same event only if both are less than $-\ln \left(\beta / d^{\prime}\right)$ or if both are greater than $\ln \left(\beta / d^{\prime}\right)$. The densities depicted in Figure 3 arise from combining the two areas that represent same events into one density and the two remaining areas that represent different events into the other density, thereby collapsing the two-dimensional space into a one-dimensional representation.

\section{Three Families of Isobias Functions for the Independent-Observations Strategy}

Isobias functions for $c_{i}$. Figure $4 \mathrm{~A}$ illustrates the isobias curves for several values of $c_{i}$. The parametric equations are provided in the Appendix, Equation A6. These isobias curves, unlike those for $c_{d}$ of the differencing strat- egy, are symmetric about the negative diagonal of the ROC square.

Isobias function for $c_{i}^{\prime}$. Figure 4B illustrates the isobias curves for several values of the relative index, $c_{i}^{\prime}$, which is simply $c_{i} / d^{\prime}$. The parametric equations are given by Equation A7 in the Appendix.

Isobias functions for $\beta_{i}$. Lastly, Figure $4 \mathrm{C}$ illustrates isobias curves for the likelihood ratio index, $\beta_{i}$, based on the independent-observations strategy. Parametric equations for these functions are given in Equation A8 of the Appendix. Again, the curves are symmetrical about the negative diagonal of the ROC square and similar (although not equivalent) in shape to the more familiar yes-no likelihood ratio functions.

\section{AN EXPERIMENTAL EXAMPLE}

To illustrate the application of these indices for the same-different design, we will report an investigation of the response biases adopted by normal people in judging whether two images of a face were the same or different. Unlike the procedure in a typical study of patients with prosopagnosia, our procedure asked observers to rate their confidence, rather than make a binary same-different decision, about the difference between the two images. We did this in order to obtain a set of isobias curves from a single experiment.

\section{Method}

Subjects and Materials. Three students (2 women and 1 man) served as observers. They viewed two color images of the same face 
A

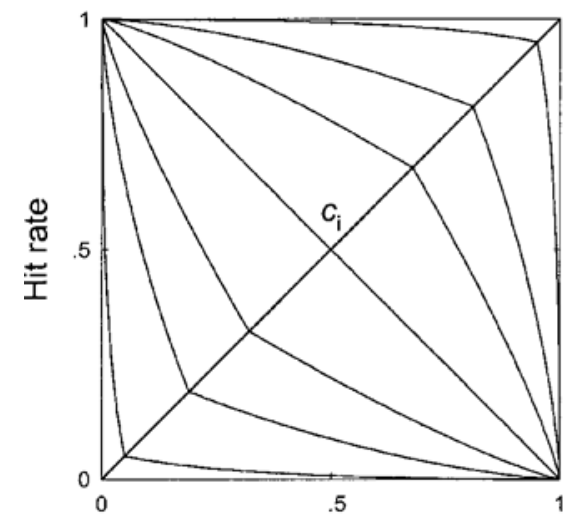

False-alarm rate
B

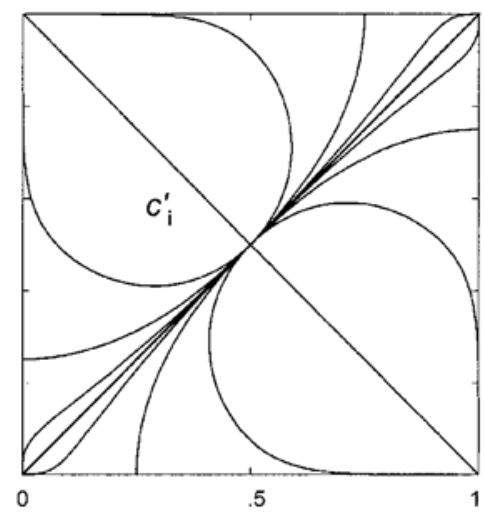

False-alarm rate

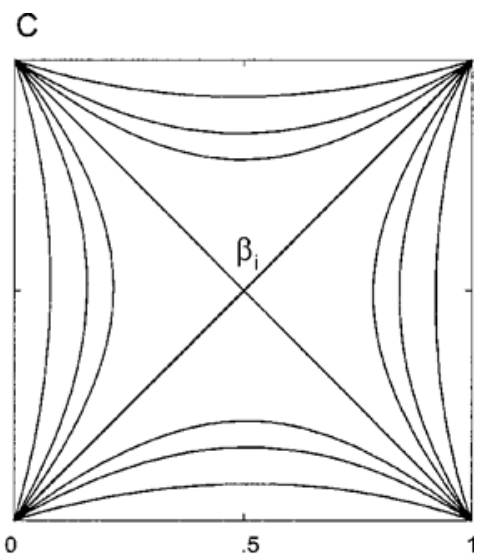

False-alarm rate

Figure 4. Isobias curves for the independent-observations strategy for $c_{i}, c_{i}^{\prime}$, and $\beta_{i}$ are shown in panels A, B, and C. The values illustrated are $c_{i}$ or $c_{i}^{\prime}=1.0,0.5,0.25,0.0,-0.25,-0.5$, and -1.0 , and $\beta_{i}=2.5,1.5,1.25,1.0,0.8,0.67$, and 0.4 . Like the functions for the differencing strategy, they are shown extending below the major diagonal.

selected from the Psychological Image Collection of Stirling University (http://pics.psych.stir.ac.uk). The images were displayed side by side on a 15 -in. monitor $(800 \times 600$ pixels $)$. The two images could be either identical or slightly different. To create different images of the same face, one of them was digitally distorted (Jasc Software) by a "pinch" deformation which moved pixels toward the center of the image. Four degrees of deformation were examined: pinchings of $6 \%, 8 \%, 10 \%$, and $12 \%$.

Procedure. An experimental session consisted of four blocks of 64 trials (16 practice trials followed by 48 experimental trials), with one block for each degree of deformation. The undistorted image (the standard) remained fixed throughout. On every trial the two images were presented for $1,500 \mathrm{msec}$. A 6-point rating scale then appeared and remained visible until the observer clicked on a number to express a confidence rating that the two images were the same $(4,5$, or 6$)$ or different $(1,2$, or 3$)$. The observer was then informed whether the two images were, in fact, the same or different. There was an equal probability that the two images would be the same or different and that the distorted image would be to the left or the right of the undistorted image. Each observer undertook two sessions to yield 96 judgments (not counting practice ones) about each percentage deformation.

Analysis. The data from each observer, and the pooled data of all the observers, each provided 20 false alarm and hit rate pairs. These constituted four ROC curves (five points each) and five isobias curves (four points each). Analysis involved a hierarchical modelfitting process that required two steps.

First, an estimate of $d^{\prime}$ was obtained for each ROC curve by maximizing the likelihood that the data arose from the model. Bias parameters were permitted to be different across the four ROC curves, and separate estimates of $d^{\prime}$ were obtained for each of the two decision strategies, differencing and independent observations. In the second step, isobias curves, based on each bias index under investigation, were fitted to the data. Again maximum-likelihood estimation was employed to estimate the values of the five bias parameters. However, the sensitivity parameters were now fixed at the values estimated in the previous step. The fixed values of $d^{\prime}$ were always those derived from the same decision strategy as the bias index that was being fitted.

The ability of the various bias indices to account for the data was assessed by calculating a chi-square statistic across the five bestfitting isobias curves for a given index. This statistic was distributed with 35 degrees of freedom $(2 \times 20$ normal deviates less 5 parameters fitted). This resulted in a chi-square statistic based on either the differencing strategy or the independent-observations strategy for each bias index.

\section{Results and Discussion}

We pooled the ratings of the 3 observers. (The results of individual observers sometimes contained empty cells that caused the analyses to fail.) Even so, the ROC analyses did not decisively favor one strategy over the other, although the independent-observations strategy gave the slightly better fit overall (Table 1). For the differencing strategy, the summed chi-square for the four ROCs gave $p\left(\chi^{2}(16)>\right.$ $28.96)=.024$; for the independent-observations strategy, the summed value gave $p\left(\chi^{2}(16)>25.94\right)=.055$. The estimated $d^{\prime}$ s for the four degrees of deformation for the differencing and independent-observations strategies are also shown in Table 1. Evidently, deformations of $10 \%$ and $12 \%$ were equally discriminable from the standard.

The goodness-of-fit statistics for eight different bias indices are shown in Table 2 . Only $c_{i}$ for the independentobservations strategy was not significantly different from the data, although $c_{d}$ for the differencing strategy fitted moderately well. Figure 5 shows how the isobias func-

Table 1

Best-Fitting $\boldsymbol{d}^{\prime}$ for Each Deformation (\%) and Goodness of Fit $\left(\chi^{2}\right.$ With 4 Degrees of Freedom $[d f]$ for Each Receiver-Operating Characteristic and $16 d f$ for the Sum) for Each Decision Strategy

\begin{tabular}{cccccc}
\hline & \multicolumn{2}{c}{ Differencing } & & \multicolumn{2}{c}{ Independent Observations } \\
\cline { 2 - 3 } \cline { 5 - 6 } Deformation & $d^{\prime}$ & $\chi^{2}$ & & $d^{\prime}$ & $\chi^{2}$ \\
\hline 6 & 1.94 & 4.31 & & 1.56 & 4.07 \\
8 & 2.98 & 7.41 & & 2.36 & 5.18 \\
10 & 3.50 & $11.29^{*}$ & & 2.75 & 5.66 \\
12 & 3.47 & 5.95 & & 2.71 & $11.03^{*}$ \\
Summed $\chi^{2}$ & & $28.96^{*}$ & & 25.94 \\
\hline
\end{tabular}

$* p<.05$. 
Table 2

Goodness of Simultaneous Fit ( $\chi^{2}$ With 35 Degrees of Freedom) to Five Isobias Curves for Each of Eight Bias Indices

\begin{tabular}{lrrrrrr}
\hline & \multicolumn{6}{c}{ Parameter Estimates } \\
\cline { 2 - 6 } Index & \multicolumn{1}{c}{1} & \multicolumn{1}{c}{2} & \multicolumn{1}{c}{3} & \multicolumn{1}{c}{4} & \multicolumn{1}{c}{$\chi^{2}$} \\
\hline$k$ & 3.49 & 2.65 & 1.78 & 0.95 & 0.31 & $72.04 \ddagger$ \\
$c_{d}$ & -1.53 & -0.71 & 0.14 & 0.94 & 1.55 & $51.55 *$ \\
$c_{d}^{\prime}$ & -0.49 & -0.21 & 0.09 & 0.40 & 0.74 & $90.80 \ddagger$ \\
$c_{s d}$ & -2.04 & -1.24 & -0.42 & 0.34 & 0.90 & $77.54 \ddagger$ \\
$\beta_{d}$ & 0.10 & 0.28 & 0.71 & 1.55 & 2.53 & $764.95 \ddagger$ \\
$c_{i}$ & -0.95 & -0.47 & 0.02 & 0.60 & 1.34 & 40.38 \\
$c_{i}^{\prime}$ & -0.40 & -0.20 & 0.01 & 0.26 & 0.56 & $60.72 \dagger$ \\
$\beta_{i}$ & 0.17 & 0.44 & 1.00 & 2.69 & 13.33 & $225.74 \ddagger$ \\
\hline
\end{tabular}

${ }^{*} p<.05 ;{ }^{\dagger} p<.01 ;{ }^{\ddagger} p<.001$.

tions for both these indices fit the data. The analyses of both accuracy and bias agree in favoring the interpretation that the observers adopted the independent-observations strategy when making these judgments. As Macmillan and Creelman (1991) have noted, the independent-observations strategy is available only when the standard is fixed (as it is here). Furthermore, the multidimensional nature of the stimuli may favor the adoption of the independentobservations strategy (Irwin \& Francis, 1995).

\section{DISCUSSION}

\section{Empirical Considerations}

It may seem prosaic to recommend the index that best describes an observer's response biases as the one to be preferred. However, that index may differ for different tasks and for different observers, and so the fact that $c_{i}$ for the independent-observations strategy, for example, best fits the data we have presented does not necessarily commend it for other cases. Indeed, some implementations of the same-different experiment, such as the roving design (see Macmillan \& Creelman, 1991), force the adoption of a differencing strategy, in which case some index other than $c_{i}$ is needed. And if, for whatever reason, the observer chooses to adopt different criteria when faced with discriminations of different degrees of difficulty, no one index may prove entirely satisfactory.

\section{Theoretical Considerations}

Macmillan and Creelman (1990) have provided a valuable assessment of the theoretical status of indices of response bias for the single-interval experiment. Here we consider some theoretical matters as they bear on the same-different experiment.

Independence from discriminability. One theoretical perspective holds that a good index of response bias should remain independent of discriminability, just as a good index of discriminability should be independent of response bias. However, as Macmillan and Creelman (1990) have put it, discriminability and bias are not symmetric in this respect. Nevertheless, investigators have sought experimental evidence for a bias measure that does not alter when discriminability alters, presumably on the assumption that observers should maintain the same bias for different degrees of discriminability (e.g., Dusoir, 1983; See, Warm, Dember, \& Howe, 1997; Snodgrass \& Corwin, 1988). This quest perhaps overlooks the fact that choice of the criterion is at the observer's disposal: There may be good or bad reasons for shifting one's criterion when faced with easy or difficult tasks. But to sup-
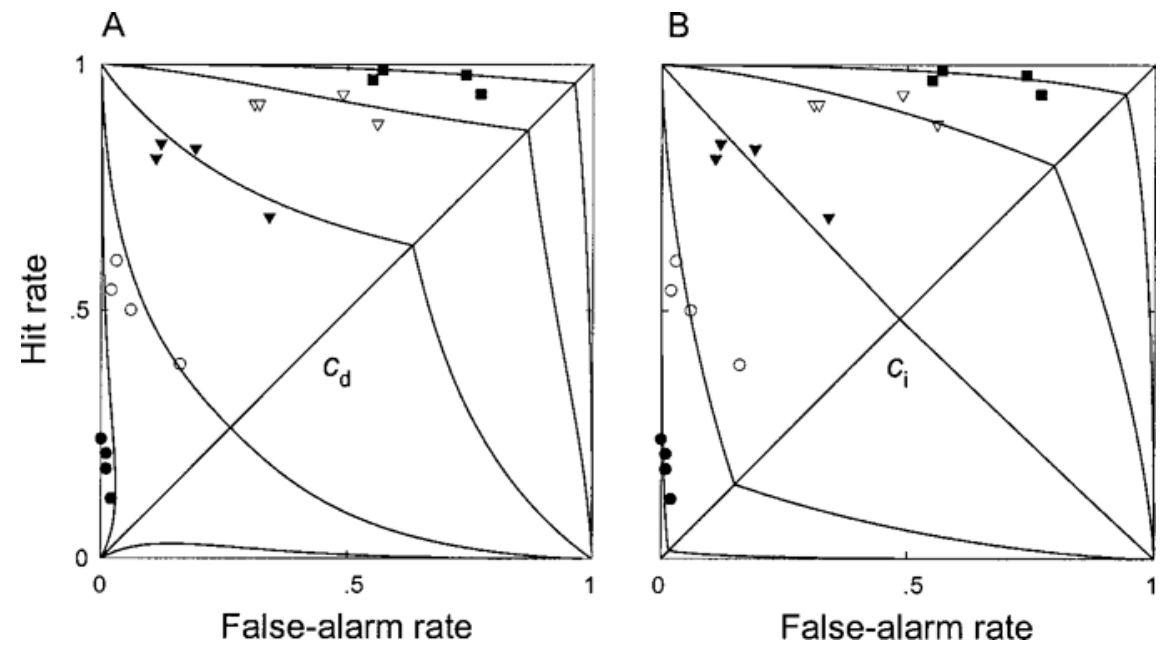

Figure 5. Best-fitting isobias functions for discriminating between two images of the same face. The different symbols represent ratings of 2 (filled squares, top) to 6 (filled circles, bottom). Panel A shows the best-fitting functions for the index $c_{d}$ of the differencing strategy, and panel $B$ shows the best-fitting functions for the index $c_{i}$ of the independent-observations strategy. The maximum-likelihood estimates of the parameters and their goodness of fit for these two indices are shown in Table 2. 
pose that discriminability might alter with response bias would be to contravene a fundamental tenet of detection theory-namely, that discriminability depends solely on the sensitivity of the observer and the parameters of the stimuli. No detection-theoretic precept requires that response bias be invariant with discriminability; hence, none of the same-different indices we have presented should be excluded on those grounds.

Range of possible values. A second characteristic proposed for a good index of response bias is that its range of possible values should be independent of discriminability. In this respect, the range of both $\beta_{d}$ and $c_{d}$ for the differencing strategy depend on $d^{\prime}$. The upper bound of $\beta_{d}$ is given by $\exp \left(d^{\prime 2} / 4\right)$, as can be seen by solving Equation A2 for $\beta_{d}$ when $k=0$. Hence, the ROC for the differencing strategy approaches the point $(0,0)$ with a finite slope of $\exp \left(d^{\prime 2} / 4\right)$. Of course, although an index like $\beta_{d}$ may have a finite range, it can still give rise to all possible points on the ROC. Similarly, the upper bound for $c_{d}$ approaches $\sqrt{2}$ as $d^{\prime}$ approaches zero. These two indices, therefore, do not possess the desirable property of their ranges' being independent of accuracy.

Monotonicity. A third characteristic proposed by Macmillan and Creelman (1990) for any satisfactory measure of response bias is that it be monotonic with the sum of the hit rate and the false alarm rate (see their Equation 2). They observed that the likelihood ratio fails this test. However, the fact that the likelihood ratio is the criterion that maximizes several important objectives (see Green $\&$ Swets, 1966; Neyman \& Pearson, 1933) gives it a special theoretical status. Macmillan and Creelman (1990) acknowledged the theoretical importance of the likelihood ratio index but argued that other measures might prove more attractive in practice. Note that for our data, the two best-fitting indices satisfy the monotonicity condition.

\section{Some Other Comparisons}

The index $k$ for the differencing strategy arising from adopting a fixed hit rate is strictly parallel to a criterion available in the yes-no experiment. In the yes-no experiment, a fixed false alarm rate is more commonly considered, in which case the observer is known as a NeymanPearson observer (see, e.g., Egan, 1975, p. 23). Because the definition of hits and false alarms is arbitrary for the same-different experiment, a constant hit rate or false alarm rate is a matter of definition.

A property of the index $\beta_{d}$ for the differencing strategy, shown in Figure $2 \mathrm{~B}$, is that the isobias curve for $\beta_{d}=1$ does not lie, as might be expected, on the negative diagonal of the ROC square, as it does for the independentobservations strategy and for the standard analysis of the yes-no experiment. This displacement of $\beta_{d}$ is also evident from the asymmetry of the ROCs. As a result, the point at which the slope of the ROC is unity and, therefore, $\beta_{d}=1$ (see, e.g., Macmillan \& Creelman, 1991) falls above the negative diagonal. Paradoxically, perhaps, an unbiased likelihood ratio observer who adopts the differencing strategy will therefore be biased toward judgments of same.

Examining the probability density functions for the differencing strategy reveals the reason for the discrepancy between $c_{d}$ and $c_{s d}$, the index derived by Macmillan and Creelman (1991, Equation 6.5). Their calculation of $c_{s d}$ for the differencing strategy is based on a common depiction of the decision space of the same-different experiment in which three underlying densities, rather than two, are portrayed. In this depiction, a central Gaussian density function for the two combinations of same events is flanked by two Gaussian densities for the two possible arrangements of the different events (see their Figure 6.4). The discrepancy between the isobias functions for $c_{d}$ and $c_{s d}$ (compare Figures $2 \mathrm{C}$ and $2 \mathrm{E}$ ) arises because the point corresponding to $d^{\prime} / 2$ for this strategy does not coincide with the neutral likelihood ratio of 1 (see Figure 1), as the three-function depiction might suggest.

\section{SUMMARY AND CONCLUSIONS}

Whenever response bias in same-different judgments needs to be measured, a variety of indices is available; we have reviewed eight. These indices are analogous, but not numerically identical, to those for the yes-no experiment. Hence, special formulae are needed for their calculation. We remain agnostic about which index is to be preferred. If but one level of accuracy is available, any index will do, but if more than one is available, the most instructive account of the observer's response biases may be that index whose isobias function best fits the data.

\section{REFERENCES}

Dusorr, T. (1983). Isobias curves in some detection tasks. Perception \& Psychophysics, 33, 403-412.

EgAN, J. P. (1975). Signal detection theory and ROC analysis. New York: Academic Press.

Gauthier, I., Behrmann, M., \& Tarr, M. J. (1999). Can face recognition really be dissociated from object recognition? Journal of Cognitive Neuroscience, 11, 349-370.

Green, D. M., \& Swets, J. A. (1966). Signal detection theory and psychophysics. New York: Wiley.

Hautus, M. J., Irwin, R. J., \& Sutherland, S. (1994). Relativity of judgements about sound amplitude and the asymmetry of the samedifferent ROC. Quarterly Journal of Experimental Psychology, 47A, 1035-1045.

Irwin, R. J., \& Francis, M. A. (1995). Perception of simple and complex visual stimuli: Decision strategies and hemispheric differences in same-different judgments. Perception, 24, 787-809.

IRWIN, R. J., \& HaUTUS, M. J. (1997). Likelihood-ratio decision strategy for independent observations in the same-different task: An approximation to the detection-theoretic model. Perception \& Psychophysics, 59, 313-316.

Irwin, R. J., Hautus, M. J., \& Butcher, J. C. (1999). An area theorem for the same-different experiment. Perception \& Psychophysics, 61, 766-769.

Macmillan, N. A., \& Creelman, C. D. (1990). Response bias: Characteristics of detection theory, threshold theory, and "nonparametric" indexes. Psychological Bulletin, 107, 401-413. 
Macmillan, N. A., \& Creelman, C. D. (1991). Detection theory: A user's guide. Cambridge: Cambridge University Press.

Macmillan, N. A., \& Creelman, C. D. (1996). Triangles in ROC space: History and theory of "nonparametric" measures of sensitivity and response bias. Psychonomic Bulletin \& Review, 3, 164-170.

Neyman, J., \& Pearson, E. S. (1933). On the problem of the most efficient tests of statistical hypotheses. Philosophical Transactions of the Royal Society of London: Series A, 231, 289-337.

NoreEn, D. L. (1981). Optimal decision rules for some common psychophysical paradigms. In S. Grossberg (Ed.), Mathematical psychology and psychophysiology (SIAM-AMS Proceedings, Vol. 13, pp. 237279). Providence: RI: American Mathematical Society.

See, J. E., Warm, J. S., Dember, W. N., \& Howe, S. R. (1997). Vigilance and signal detection theory: An empirical evaluation of five measures of response bias. Human Factors, 39, 14-29.
Snodgrass, J. G., \& Corwin, J. (1988). Pragmatics of measuring recognition memory: Applications to dementia and amnesia. Journal of Experimental Psychology: General, 117, 34-50.

TArr, M. J., Gauthier, I., \& Behrmann, M. (1998, April). Measuring prosopagnosicpatients' recognition sensitivity and bias for faces and objects using ROC curves. Poster session presented at the annual meeting of the Cognitive Neuroscience Society, San Francisco.

\section{NOTE}

1. The definition of hits and false alarms is arbitrary for the samedifferent experiment. Following Noreen (1981), we define a hit as calling two events the same when they were in fact the same and a false alarm as calling two events the same when they were in fact different.

\section{APPENDIX}

This appendix presents the parametric equations, of the form $F=f_{1}(s)$ and $H=f_{2}(s)$, for the functions illustrated in the figures. The first term, $F$, is the false alarm rate for the model, and the second term, $H$, is the hit rate. With the isobias index as the parameter, the equations generate the correspondingisobias function; with $d^{\prime}$ as the parameter, they generate the corresponding ROC. The equations give the isobias and ROC curves for nonnegative values of $d^{\prime}$; the curves for negative values of $d^{\prime}$ can be obtained by reflection about the major diagonal.

\section{Differencing Strategy}

1. Parametric equations for isobias functions based on a particular value of the decision axis, $k$ (see Hautus et al., 1994, Equations 1 and 2) are as follows:

$$
F=\Phi\left(\frac{k+d^{\prime}}{\sqrt{2}}\right)+\Phi\left(\frac{k-d^{\prime}}{\sqrt{2}}\right)-1, \quad H=2 \cdot \Phi\left(\frac{k}{\sqrt{2}}\right)-1,
$$

where $\Phi(\bullet)$ is the cumulative normal distribution function.

For each of the remaining indices of bias we first determine the relationship between the value of the index and the value of the decision axis. The particular equation that relates each index to the decision axis can then be substituted for $k$ in Equation A1 to give the parametric equations for isobias functions based on each index.

2. Parametric equations for isobias functions based on the likelihood ratio for the differencing strategy, $\beta_{d}$, are given by the ratio of the likelihoods of same to different events. The density function for same events is a normal distribution, with a mean of zero; that for different events is half the sum of two normal distributions, with means of $d^{\prime}$ and $-d^{\prime}$. The ratio of these two densities is

$$
\beta_{d}=\left(e^{-d^{\prime 2} / 4} \frac{1}{2}\left(e^{d^{\prime} k / 2}+e^{-d^{\prime} k / 2}\right)\right)^{-1} .
$$

Note that because

$$
\begin{gathered}
\cosh (z)=\frac{1}{2}\left(e^{z}+e^{-z}\right), \\
\beta_{d}=\left(e^{-d^{\prime 2} / 4} \cosh \left(d^{\prime} k / 2\right)\right)^{-1} .
\end{gathered}
$$

Finally, solve for $k$ :

$$
k=\frac{2}{d^{\prime}} \operatorname{arcosh}\left(\frac{1}{\beta_{d}} e^{d^{2} / 4}\right),
$$

where $\operatorname{arcosh}(\bullet)$ is the inverse hyperbolic cosine function. The parametric equations for the index $\beta_{d}$ are obtained by substituting Equation A2 into Equation A1.

3. To obtain parametric equations for isobias functions based on $c_{d}$, we must start with Equation A2, because $c_{d}$ is defined as the distance on the decision axis from $\beta_{d}=1$. So the parametric equations for the index $c_{d}$ are obtained by substituting into Equation A1.

$$
k=\frac{2}{d^{\prime}} \operatorname{arcosh}\left(e^{d^{\prime 2} / 4}\right)-c_{d}
$$

4. Parametric equations for isobias functions based on $c_{d}^{\prime}$ are obtained from the definition $c_{d}^{\prime}=c_{d} / d^{\prime}$. Thus, $c_{d}=c_{d}^{\prime} d^{\prime}$ can be substituted into Equation A3 to yield

$$
k=\frac{2}{d^{\prime}} \operatorname{arcosh}\left(e^{d^{2} / 4}\right)-c_{d}^{\prime} d^{\prime},
$$

which, in turn, is substituted into Equation A1 to obtain the parametric equations based on the index $c_{d}^{\prime}$.

5. Parametric equations for isobias functions based on $c_{s d}$ are obtained by substituting the equation

$$
k=\frac{d^{\prime}}{2}-c_{s d}
$$

in Equation A1. Note that this definition is different from that offered by Macmillan and Creelman (1991, Equation 6.5), because we have defined a hit as the probability of making the response same when the events are the same.

\section{Independent-Observations Strategy}

Because of a discontinuity in the first derivative of the density functions for the independent-observations strategy (at $c_{i}=$ 0 or $\beta_{i}=1$ ), the complete isobias functions for this strategy are defined as continuous piecewise using the step function, where $\operatorname{step}(x)=1$ for $x \geq 0$ and $\operatorname{step}(x)=0$ for $x<0$. For compactness, some of the variables are first defined separately. 


\section{APPENDIX (Continued)}

6. Parametric equations for isobias functions based on $c_{i}$ were first reported by Irwin and Hautus (1997; Hautus et al., 1994), but in a form not easily recognizable in the present context. If we let $\ln (\beta) / d^{\prime}=c_{i}$ in Equations 5 and 6 of Irwin and Hautus (1997) and combine the equations for both halves of the ROC curve, the following parametric equations are obtained for $c_{i}$ of the independent-observations strategy:

$$
\begin{aligned}
& t_{1}=2 \cdot\left(\Phi\left(c_{i}-\frac{d^{\prime}}{2}\right)-1\right) \cdot\left(\Phi\left(c_{i}+\frac{d^{\prime}}{2}\right)-1\right) \\
& t_{2}=\left(\Phi\left(c_{i}-\frac{d^{\prime}}{2}\right)-1\right)^{2}+\left(\Phi\left(c_{i}+\frac{d^{\prime}}{2}\right)-1\right)^{2} \\
& F=\operatorname{step}\left(c_{i}\right) \cdot t_{1}\left(c_{i}, d^{\prime}\right)+\operatorname{step}\left(-c_{i}\right) \cdot\left[1-t_{2}\left(-c_{i}, d^{\prime}\right)\right] \\
& H=\operatorname{step}\left(c_{i}\right) \cdot t_{2}\left(c_{i}, d^{\prime}\right)+\operatorname{step}\left(-c_{i}\right) \cdot\left[1-t_{1}\left(-c_{i}, d^{\prime}\right)\right] .
\end{aligned}
$$

7. Parametric equations for isobias functions based on $c_{i}^{\prime}$ are obtained using the definition $c_{i}^{\prime}=c_{i} / d^{\prime}$, as for the case of the differencing strategy. Substitution of $c_{i}=c_{i}^{\prime} d^{\prime}$ in Equation A6 yields

$$
\begin{aligned}
& F=\operatorname{step}\left(c_{i}^{\prime}\right) \cdot t_{1}\left(c_{i}^{\prime} d^{\prime}, d^{\prime}\right)+\operatorname{step}\left(-c_{i}^{\prime}\right) \cdot\left[1-t_{2}\left(-c_{i}^{\prime} d^{\prime}, d^{\prime}\right)\right] \\
& H=\operatorname{step}\left(c_{i}^{\prime}\right) \cdot t_{2}\left(c_{i}^{\prime} d^{\prime}, d^{\prime}\right)+\operatorname{step}\left(-c_{i}^{\prime}\right) \cdot\left[1-t_{1}\left(-c_{i}^{\prime} d^{\prime}, d^{\prime}\right)\right],
\end{aligned}
$$

where $t_{1}$ and $t_{2}$ are as defined above.

8. Parametric equations for isobias functions based on $\beta_{i}$ are presented by Irwin and Hautus (1997, Equations $2-4)$. We again present these functions, but in a more compact form. First define the equations:

$$
\begin{aligned}
& t_{3}\left(d^{\prime}, \beta_{i}\right)=\int_{\ln \beta_{i} / d^{\prime}}^{\infty} \int_{y\left(x, \beta_{i}\right)}^{\infty} f_{-d^{\prime} / 2,-d^{\prime} / 2}+f_{d^{\prime} / 2, d^{\prime} / 2} d y d x \\
& t_{4}\left(d^{\prime}, \beta_{i}\right)=\int_{\ln \beta_{i} / d^{\prime}}^{\infty} \int_{y\left(x, \beta_{i}\right)}^{\infty} f_{-d^{\prime} / 2, d^{\prime} / 2}+f_{d^{\prime} / 2,-d^{\prime} / 2} d y d x,
\end{aligned}
$$

where $f_{\mu_{x} \mu_{y}}$ is the bivariate normal density function of sensory evidence $(\sigma=1, \rho=0)$, with dimensions $x$ and $y$ representing the evidence arising from each of the two observations of a trial. The marginal means for each density function are specified by the subscripts. $t_{3}\left(d^{\prime}, \beta_{i}\right)$ is the volume under the density functions arising from same events beyond the criterion $\beta_{i}$ when $\beta_{i} \geq 1 . t_{4}\left(d^{\prime}, \beta_{i}\right)$ is the corresponding volume under the density functions arising from different events. These quantities therefore are equal to $H$ and $F$ only when $\beta_{i} \geq 1$. By taking advantage of reflection symmetry about $y=-x$ and introducing piecewise functions, the parametric equations for the isobias functions based on $\beta_{i}$ can be determined for all values of $\beta_{i}$.

$$
\begin{aligned}
& F=\operatorname{step}\left(\ln \beta_{i}\right) \cdot t_{4}\left(d^{\prime}, \beta_{i}\right)+\operatorname{step}\left(\ln \frac{1}{\beta_{i}}\right) \cdot\left(1-t_{3}\left(d^{\prime}, \frac{1}{\beta_{i}}\right)\right) \\
& H=\operatorname{step}\left(\ln \beta_{i}\right) \cdot t_{3}\left(d^{\prime}, \beta_{i}\right)+\operatorname{step}\left(\ln \frac{1}{\beta_{i}}\right) \cdot\left(1-t_{4}\left(d^{\prime}, \frac{1}{\beta_{i}}\right)\right) .
\end{aligned}
$$

